\title{
An Approach Defining Gait Recognition System using K- Means and MDA
}

\author{
Riant Kaur \\ M.Tech $\left(4^{\text {th }}\right.$ sem $)$ \\ Department of IT \\ CEC, Landran, Mohali (Punjab)
}

\author{
Heena \\ Assistant Professor \\ Department of IT \\ CEC, Landran, Mohali (Punjab)
}

\begin{abstract}
Biometric Authentication is a technology of verifying and identifying individuals. Gait Recognition is a type of biometric identification method which is related to behavioral characteristic of a person. Gait identifies a person based on the manner he moves or walks on foot. Gait needs reduced detail and is difficult to conceal Gait can identify people from a distance without their cooperation. Gait can be used in real time environments to detect threats and frauds. Gait can be captured using moving video, floor sensors or motion recording sensors. In the proposed work, firstly Gait is captured using moving video and binary silhouettes are generated. Secondly, feature extraction from each silhouette is done using GPPE. Lastly, recognition is performed using SVM, K-Means and MDA. Here, all the work will be performed on live input videos and gait MATLAB database.
\end{abstract}

\section{General Terms}

Biometric analysis, Gait, Feature extraction, identification, pattern classification and recognition.

\section{Keywords}

Gait Recognition System, Gait Pal and Pal Entropy (GPPE), Support Vector Machine (SVM), K-Means, Multi-linear Discriminant Analysis (MDA).

\section{INTRODUCTION}

User authentication is the process of verifying identity. Biometric authentications are used. Biometric is a field of technology that uses automated methods for identifying and verifying a person. In real time applications like in banks, airports, biometric identification methods are used. Biometric characteristics are of two types: When parts of human body are directly measured, the characteristics obtained from those measurements are called physiological characteristics. e.g.: palm prints, finger prints, DNA etc. These are directly related to human body. The characteristics which are related to the behavioral changes of human are known as behavioral characteristics e.g.: speech and gait.

Gait Analysis: Gait analysis is the methodical review of human locomotion, elevated by instrumentation for the purpose of measuring muscle's activity, movements and mechanics of body.

\subsection{Gait Recognition System}

1.1.1 Tracking a person by Video capture: Method of accurate tracking of person in indoor surveillance video stream obtained from static camera.

\subsubsection{Formation of binary silhouettes by} Background Subtraction: In this approach moving objects are identified from the portion of video frame that differs from the background model. Background subtraction generates binary images containing black and white (moving pixels) also known as binary silhouettes. It can be classified into 2 types:

Non-Recursive methods: These use sliding window approach for background estimation. Disadvantage of this method is memory storage requirement.eg. Median Filter

Recursive methods: Background model is recursively updated based on each input frame. Recursive techniques require less storage. eg. Gaussian Model

1.1.3 Removal of noise by Pre-processing: Preprocessing is done on video frames to reduce presence of noise.

\subsubsection{Formation of feature matrix by Feature} Extraction: Extraction of features is a special type of reduction of dimensionality. The input data is transformed into a set of features and is known as feature extraction. Feature extraction can be done by two ways:

Model based: Model based approaches aim to model human body or motion with geometrical curves.

Model free/Holistic: The holistic methods attempt to characterize the spatial variation of dynamic variables in Gai cycle.

Principal Component Analysis (PCA): PCA is used to reduce the data dimensionality of feature vector. It is used to resize the various sizes of images into same size.

1.1.5 Classification by Recognition: In this step input videos are compared with sequences stored in database Different types of classifiers are used for the recognition. The minimum distance classifier may be used for gait recognition.

\subsection{Gait Pal and Pal Entropy (GPPE)}

It measures the uncertainty associated with a random variable for each pixel in the silhouette images. It is used in feature extraction.

\subsection{Support Vector Machine (SVM)}

It is a state-of-the-art classification method to calculate and process the high-dimensional data such as gene expression, and flexibility in modeling diverse sources of data .SVMs belong to the general category of kernel methods. A kernel function can replace the dot products and the kernel method depends on the data only through these dot-products. The Kernal method computes a dot product in some possible high dimension feature space. 


\subsection{Multi-linear Discriminant Analysis (MDA)}

It is a general supervised dimensionality reduction framework which can avoid the curse of dimensionality and serves the small sample size problem as it is performed in a much lowerdimension feature space than LDA. It is applied with SVM to increase effectiveness.

\subsection{K-means Algorithm}

It is an iterative refinement heuristic algorithm that works faster. A common method is to run the algorithm several times to regain the best clustering found. K-means clustering procedures can be applied for scalable image retrieval from large databases.

\section{RELATED WORK}

Liang Wang et al. [6] proposed a Gait recognition method using the spatial-temporal silhouette analysis. To track and segment the moving silhouettes from a walking figure they used background subtraction. Then the time-varying distance signals were derived from sequence of silhouette images and they used these for dimensionality reduction of input feature space. They applied the eigenspace transformation based on Principal Component Analysis (PCA) to these distance signals, hence reducing the dimensionality of the feature space. Finally, They performed supervised pattern classification techniques in the lower-dimensional eigenspace for recognition. Their proposed method had an encouraging recognition performance with low computational cost.

Qiong Cheng et al. [10] proposed a method of gait recognition using Linear Discriminant Analysis (LDA) and Principal Component Analysis (PCA) . Firstly, they applied PCA to 1D time-varying signals of distance which are derived from the sequence of silhouette images for dimensionality reduction. Then, they applied LDA to optimize pattern classification. Then, they used Spatio-temporal Correlation (STC) and Normalised Euclidean Distance (NED) to measure two different sequences. Finally, $\mathrm{K}$ nearest neighbour classification $(\mathrm{KNN})$ is performed for recognition. Their proposed experimental results proved that gait recognition algorithm using LDA and PCA is better than the one based on only PCA.

M. Pushpa Rani et al. [8] proposed a system of gait recognition for identification of humans using Modified Independent Component Analysis (MICA). Firstly, they performed the background modeling from a video sequence and then segmented the foreground moving objects in the individual frame image using the background subtraction algorithm. Then, they used morphological skeleton operator to track all the moving silhouettes of a walking person. Finally, the MICA based on eigenspace transformation is trained using the sequence of silhouette images and recognition is performed. Their proposed algorithm achieved a pleasing recognition performance.

Principal Component Ananlysis (PCA) is used to reduce the dimensionality of the images without a large information loss. Hayder Ali et al. [3] proposed a gait recognition method in which they used Principle Component Analysis (PCA) for gait recognition purposes. They described about extraction of silhouette method using the CMU gait database and rearranged the database for purpose of identification. They tested three styles of walking fast, slow, and with a ball from the CMU MoBo gait database. They found that walking with a ball gives the $89 \%$ highest equal error rate. Some researchers represented better rates of recognition for walking in different styles and conditions.

A. Hayder et al. [1] proposed a new method for gait recognition purposes that is Principal Component Analysis (PCA) without Radon Transform and with Radon Transform (RT). The Radon Transform is used to detect features within an image and PCA is used for the reduction of dimensionality of the images without great information loss. They used Radon Transform to detect features first and PCA to reduce their dimensionality afterwards. They considered various parameters that is the side view of slow walk, side view of fast walk and carrying a ball walk. These were selected from CMU MoBo database for carrying out experiments. The two techniques experimental result achieved equal recognition rates (EER) for RT with PCA and for PCA only. Their work gave average results as better rate of recognition for different walking styles in different gait database conditions are given by some researchers.

Lili Liu et al. [5] proposed a gait recognition algorithm based on Outermost Contour. Firstly, they used adaptive silhouette extraction algorithm to segment the images. Then, they applied post processing techniques to normalize each image having less noise. After this process, they performed feature extraction based on outermost contour. Then, they used Principal Component Analysis (PCA) and Multi-linear Discriminant analysis (MDA) to reduce the data dimensionality and optimize the class separability of different gait sequences simultaneously. In the end, they used simple pattern classification for recognition process.

Sagar A. More et al. [11] gave a brief survey on gait biometrics. They concluded that Gait is a behavior characteristic that possesses individual difference formed in course of human's growth. Gait is all external appearance that consists of human body structure, motion regulating system, behavioral and psychological activities when person is walking, compared with other biometrics, gait requires no object contact and is measured at a distance. It is applied to passive vision surveillance scenario such as ranks, airports and military departments. Gait is affected by various physiological, psychological and external factors such as footwear, clothing, surface of walking, mood, illness, fatigue, and so on.

Ira Gaba et al. [4] proposed a novel and simple gait recognition method. Firstly, they converted the input video into frames and detected binary silhouette of a walking person from each frame. Secondly, they performed feature extraction using image processing operation. Using hanavan's model and CBIR method, they considered length of one hand, distance between head and feet, length of leg and distance between both hands, as key features. At last, they used Back Propagation Neural Network (BPNN) + Multi-linear Discriminant Analysis (MDA) and BPNN+ Linear Discriminant Analysis (LDA) techniques for training and testing purposes. By using the combination of LDA, BPNN and MDA, they obtained better accuracy results.

Parneet Kaur et al. [9] proposed a simple but effective method for person recognition from body silhouette and gait. Firstly, they detected binary silhouette of a walking person from each frame. Secondly, they performed the feature extraction process using image processing operation. Here, center of mass, step size length, and cycle length were taken 
as key features. At last they used Neural Network (NN) and Exemplar Neural Network (ENN) techniques for training and testing purpose. Their proposed work gave promising results but had to be improved for large data bases, clothing style conditions and for different walking considerations.

M.Jeevan et al. [7] proposed novel temporal Gait Pal and Pal Entropy (GPPE) representation for every cycle of silhouette. They used GPPE for feature extraction and Principal component analysis (PCA) to reduce the dimensionality and create a feature matrix. Then they used Support Vector Machine (SVM) for training of individuals and testing of individuals by the method being proposed. They tested the performance of the proposed feature on CASIA Gait and Treadmill Databases and fared well over Shannon entropy. Their proposed feature reduced the sensitivity of recognition towards the covariate conditions.

Arun Joshi et al. [2] proposed a method for automatic person recognition from body silhouette and gait. Firstly, they detected binary silhouette of a walking person from each frame. Secondly, they extracted features from each frame using image processing operation. They considered centre of mass, step size length, and cycle length as key features. At last they used Back Propagation Neural Network (BPNN) and Support Vector Machine (SVM) for training and testing purposes and the results are obtained.

\section{PROPOSED WORK}

(a) In the proposed Gait Recognition System, live input video will be captured and taken as input.

(b) Gait Pal and Pal Entropy (GPPE) representation will be proposed for feature extraction. The Principal component analysis (PCA) will be applied to all the extracted features to create a feature matrix.

(c) Here, new key features i.e., height, length of neck, length of leg, length of hand will be extracted and selected for recognition purposes.

(d) Support Vector Machine (SVM) will be used for training and testing of individuals. MDA will be applied in SVM, solving problems of dimensionality, small sample size and reducing the computational cost in learning stage.

(e) K-means algorithm will help in nearest neighbour search efficiently which will save time in matching process.

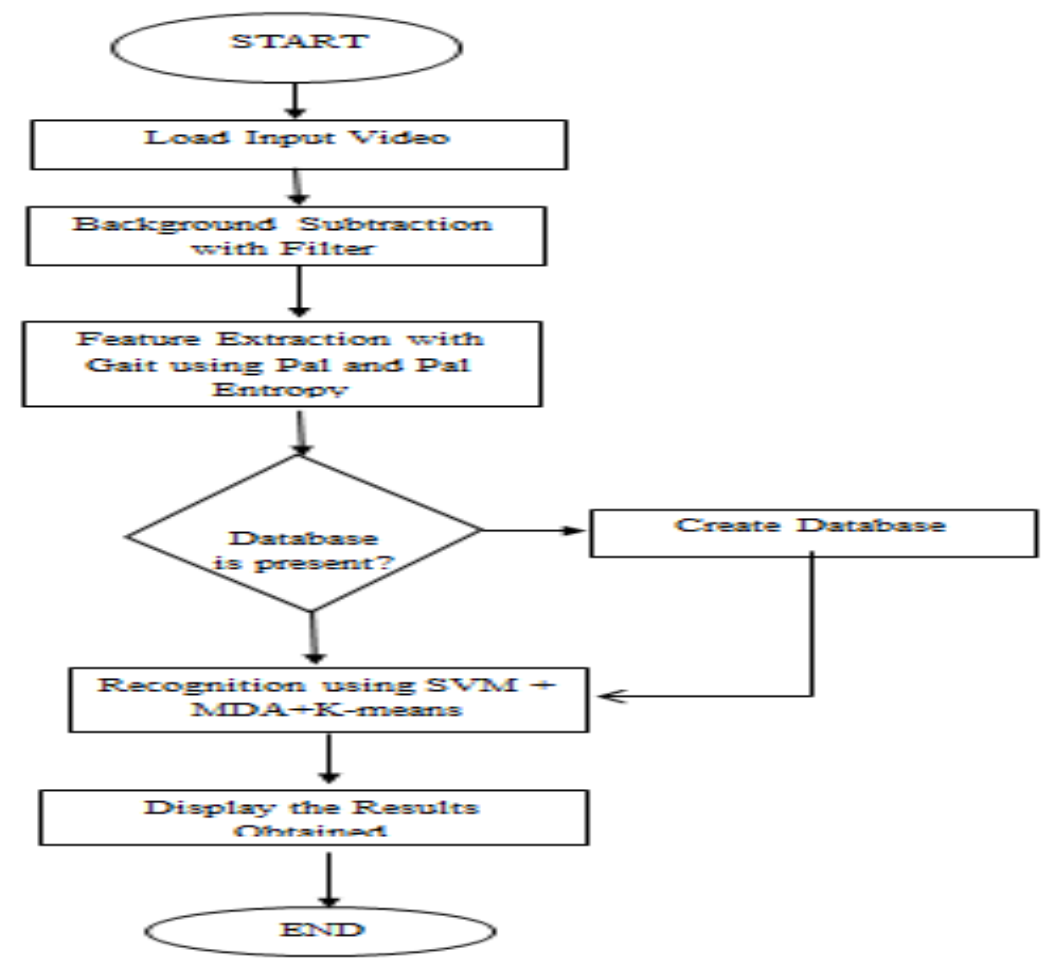

Fig 1: Flowchart of proposed method

\section{CONCLUSION}

Gait Recognition System based on Support Vector Machine (SVM), K-means algorithm and Multi-linear Discriminant Analysis (MDA) is proposed. SVM will be used to analyze data, recognize patterns and for classification purposes in recognition phase. K-means algorithm will group the images into clusters based on their color content for efficient nearest neighbor searching and MDA will work for high dimensional space sample problems by reducing the dimensionality. The proposed Gait Recognition System will be a system which will provide better identification results. The problems like low dimensionality; time consumed for classification of patterns will be solved using these techniques. Matching of input data with stored database, giving results in less time and giving accurate results, are the advantages of using this proposed system; so that the security of an organization is not hampered in any way and any security breach can be identified efficiently in future.

\section{ACKNOWLEDGMENT}

Special thanks to my Guide for helping me and guiding me throughout my disquisition. Thanks to almighty and my family members for their endless support. 


\section{REFERENCES}

[1] A. Hayder, J. Dargham, A. Chekima, and G. M. Ervin,"Person Identification Using Gait", International Journal of Computer and Electrical Engineering, V. 3, No.4,Aug 2011, pp. 477-482.Ding, W. and Marchionini, G. 1997 A Study on Video Browsing Strategies. Technical Report. University of Maryland at College Park.

[2] Arun Joshi, Shashi Bhushan, Jaspreet Kaur, "Gait Recognition of human using SVM and BPNN classifiers", International Journal of Computer Science and Mobile Computing, V. 3(1), 2014, pp.281-290. Tavel, P. 2007 Modeling and Simulation Design. AK Peters Ltd.

[3] Hayder Ali, Jamal Dargham, Chekima Ali, Ervin Gobin Moung, "Gait Recognition using principle Component Analysis", Computer Engineering Program, School of Engineering and Information Technology, University Malaysia Sabah, Kota Kinabalu, The 3rd International Conference on Machine Vision, 2010, pp. 539-543.

[4] Ira Gaba, Paramjit Kaur, "Biometric Identification on The Basis of BPNN Classifier with Other Novel Techniques Used For Gait Analysis", International Journal of Recent Technology and Engineering, V. 2(4), 2013, pp. 137-142.

[5] Lili Liu, Yilong Yin, Wei Qin and Ying Li, "Gait Recognition Based on Outermost Contour", International Journal of Computational Intelligence Systems, V. 4, No.5, Sep 2011, pp. 1090-1099.

[6] Liang Wang, Tieniu Tan, Huazhong Ning, and Weiming $\mathrm{Hu}$, "Silhouette Analysis-Based Gait Recognition for
Human Identification",IEEE Transactions on pattern analysis and machine intelligence, V.25, No.12, 2003, pp. $1505-1518$.

[7] M.Jeevan, Neha Jain, M.Hanmandlu, GirijaChetty, "Gait Recognition Based On Gait Pal and Pal Entropy Image", IEEE, 2013, pp. 4195-4199.

[8] M. Pushpa Rani and G.Arumugam,“An Efficient Gait Recognition System for Human Identification using Modified ICA", International Journal of Computer Science and Information technology (IJCSIT) V.2, No.1, 2010, pp. 55-67.

[9] Parneet Kaur, Gurjot Kaur Walia, Amandeep Singh Dhaliwal, "Gait Recognition System for Improved Human Identification using ENN and NN", International journal of Advanced Research in Computer Science and Software Engineering, V.3(11), Nov 2013, pp. 11541161.

[10] Qiong Cheng, Bo Fu, and Hui Chen, "Gait Recognition Based on PCA and LDA", Proceedings of the Second Symposium International Computer Science and Computational Technology (ISCSCT '09) Huangshan, P. R. China, 2009, pp. 124-127.

[11] Sagar A. More and Pramod J. Deore, "A Survey on Gait Biometrics", World Journal of Science and Technology, V. 2(4), 2012, pp. 146-151.

[12] Sanjeev Sharma, Ritu Tiwari, Anupamshukla and Vikas Singh, "Identification of People Using Gait Biometrics", International Journal of Machine Learning and Computing, V.1,No.4 2011, pp. 409-415 